\title{
CIRUGIA Y ORTOPEDIA EN LAS AFECCIONES NERVIOSAS DE LA INFANCIA
}

\author{
For el Dr. RICARDO J. CARITAT \\ Monterideo, Uruguav.
}

Consideraciones de carácter general.

Dentro de la Patología y la Clínica de la infancia existe un grupo de afecciones del Sistema Nervioso Central, cuyo tratamiento entra en los dominios de la Ortopedia incruenta o cruenta, según las circunstancias; para expresar con más exactitud nuestro pensamiento, diremos que sus tratamientos constituyen problemas de indole exquisitamente ortopédica y reeducadora.

El éxito o el fracaso de esa tarea ortopédica y reeducadora, ciepende en algunas de esas afecciones de la capacidad intelectual de los pacientes y de la intensidad de su trastorno motor; en otras depende. en cambio, exclusivamente, de la 1esión del aparato locomotor. A este último grupo pertenecen la parálisis infantil y las parálisis obstétricas; al primero la compleja agrupación de manifestaciones patológicas que las escuelas ortopédicas norteamericanas llaman genéricamente espásticos y que incluye las hemiplegias infantiles, las paraplegias y los tipos verdaderamente generalizados, en los cuates bay espasticidad, no solamente de los cuatro miembros, sino que todo el paciente es en conjunto un rígido; a éstos corresponde bien el término de espásticos en su acepción genérica $y$ son ellos los que plantéan los problemas más difíciles de tratamiento.

En el grupo de los espásticos, las posibilidades de reeduración dependen en parte considerable de su capacidad intelectual y los métodos reconstructivos son de segundo otden: en rambio, en la parálisis infantil y las parálisis obstétricas, las posibilidades de reeducación y de rehabilitación están basadas exclusivamente en las posibilidades de aplicación de los métodos de reconstrucción; éstos son, pues, aquí de primer plano. 
Para todos los pacientes de estos diversos grupos, la reeducación es de importancia primordial y debe decirse que nada puede obtenerse sin un programa reeducacional bien planeado, de acuerdo con las posibilidades de realización motoras e intelectuales: que es necesario un guía poseedor de conocimientos amplios de la fisiopatología del movimiento, de las acciones aisladas $y$ agrupadas, de los sincronismos $y$ asincronismes: de los agonismos y los antagonismos musculares; es necesario conocer también las posibilidades de adaptación y de sustitución muscular, la fisiopatología de las actitudes viciosas y de todos los métodos a oponer para evitar su transformación en deformación fija y conocer también los métodos de corrección y de estabilización. Es necesario, además, estar pronto para medificar las indicaciones de acuerdo con las necesidades y poseer un tesón y una persistencia vecinas de 10 cargoso para obtener resultados apreciables.

A todo ello hay que agregar un complemento fisioterápico bien seleccionado, bien instituído, mejor realizado, des . terrándose los métodos tipo standard para tratar de llenar las necesidades individuales.

\section{A) Los espásticos.}

En este amplio grupo de pacientes, dijimos que las posibilidades de éxito dependen fundamentalmente de la capacidad intelectual: existe. además, un segundo factor de gran importancia y es la participación en el cuadro del sistema extrapiramidal, participación que puede ser nula o más o menos extensa y más o menos profunda.

La capacidad intelectual y el sistema extrapiramidal plantean problemas de reeducación de gran complejidad y dificultad y ortopédicamente sólo podemos actuar contra la espasticidad de origen piramidal.

En todos estos pacientes debe instituirse desde tierna edad un correcto y mantenido tratamiento profiláctico de las actitudes viciosas y las deformaciones, lo cual significa oponer una resistencia pasiva a las contracturas. Ese tratamiento pro. filáctico consistirá en el uso de aparatos amovibles, apropia. dos, livianos. fáciles de higienizar, que serán quitados para intercalar secciones de movilización y estiramientos pasivos $y$ masaje tipo sedante. Más adelante, cuarido la edad, la comprensión y las posibilidades de realización del niño lo permitan. las sesiones de estiramiento y movilización pasiva serán completadas por la movilización activa, el ejercicio activo de 
los músculos en déficit, dejando en reposo los músculos en espasmo; de este modo, el niño irá progresivamente aprendiendo a relajar, a distender sus músculos en contractura $y$, por consiguiente, a ejercitar los antagonistas de los músculos generadores de las actitudes viciosas.

Paralelamente al tratamiento del déficit del aparato 10comotor debe instituirse, en cuanto la edad del niño lo permita, el tratamiento de su intelecto o de su déficit intelectual. El despertar de la inteligencia es absolutamente necesario, porque sin comprensión y sin cooperación de parte del paciente no es posible obiener ninguna mejoría del trastorno motor.

Se les debe enseñat a hablar, leet y escribir, sentarse, ccmer, terapia educacional y efectuando todo ello, jugando dentro de lo posible, con profusión de figuras e imágenes en colores, en pequeños grupos de niños y buscando la emulación. Desde este punto de vista son ejemplares las instituciones norteamericanas de ese género, como por ejemplo, la Jesse Espalding School de Chicago. Consideramos que este tratamiento que hemos expuesto en sus lineamientos generales es extraordinariamente importante, tanto que constituye el fundamento, la base del tratamiento de los espásticos.

Por razones que no hemos de entrar a considerar en este momento, esa parte del tratamiento, la Ortopedia incruenta y preventiva y la reeducación, es precisamente la gran laguna a llenar en estos países de América Latina. El gran obstáculo que encuentra en eí camino todo aquél que se interese por el triste porvenir de estos niños, es la falta de un establecimiento organizado, en el cual el niño reciba de parte de un persona! especializado. los cuidados y las enser̃anzas necesarias para su rehabilitación total o parcial, de acuerdo con las posibilidades. Entre nosotros y gracias a la iniciativa privada, comienzan a darse los primeros pasos en ese sentido, con la instalación de una pequeña escuela, en la cual esos niños serán beneficiados con un doble tratamiento paralelo; al del déficit intelectual y el del déficit motor mediante la reeducación.

Cuando esta última bien instituída, bien realizada durante prolongados períodos, se muestre parcialmente insuficiente para remediar determinadas impotencias funcionales o para la corrección de ciertas actitudes viciosas, en cierto tipo de niños muy bien seleccionados, con muy buena mentalidad, vecina de lo normal, de carácter más bien apacible. se recurrirá a la Ortopedia cruenta, quirúrgica. Entendemos que se debe ser muy circunspecto, muy estricto, muy exigente para plantear indicaciones operatorias en los espásticos y que en los irritables 
y en aquéllos en que el sistema extrapiramidal tiene una importante participación, la cirugía está contraindicada (hablamos en términos generales), porque el dolor y el temor agrava en ellos el estado de irritabilidad y de contractura.

Repetimos, pues, que la cirugía o la Ortopedia quírúrgica están indicadas en los espásticos. de carácter apacible. exclusivamente piramidales o con poca participación de lo extrapiramidal, con muy buena mentalidad, luego de un intenso y prolongado período de reeducación que nos muestre sus posibilidades y todavía con el agregado de que se realizarán en ellos intervenciones 10 más fáciles posibles desde el punto de vista récnico y de resultados seguros.

Si el tratamiento profiláctico de las deformaciones y posiciones viciosas no ha sido instituído o si fué mal realizado y las deformaciones se han producido, es necesario corregirias y para él disponemos, en primer lugar, de los métodos incruentos; yesos en varias etapas con o sin narcosis. método del torniquete, etc., y si ellos fracasaran o no estuvieran indicados. se pasará a la etapa quirúrgica, de la cual nos limitamos a exponer los conceptos generales.

Pie equino: Se recurre a la operación de Stoffel con resección de las ramas de inervación de los gemelos y el soleo, o al alargamiento del tendón de Aquiles, según las circunstancias, es decir, según que existe o no acortamiento del tendón. Cuando los músculos dorsales del pie muestren muy escaso poder contráctil y con más razón aun cuando estén francamente anulados, será necesaric agregar una artrorisis posterior para evitar la recidiva del equinismo. Y cuando al equinismo se agreguen desplazamientos laterales en el sentido del varo o del valgo, con pobre musculatura para restaurar el equilibrio lateral, la intervención ósea a agregar al alargamiento del tendón de Aquiles. será la artrodesis sub-astragalina y medio tarsiana, con artrorisis posterior de la tibio tarsiana. Naturalmente que este plan operatorio debe ser cumplido en varias etapas.

Flexión de la rodilla: Alargamiento de los tendones flexores para las flexiones que se resisten a los métodos conservadores; combinado a capsulotomía posterior para los casos más severos a si no se efectuará una osteotomía supracondílea.

El trasplante del tensor de fascia lata a la rótula o en casos más serios, el bíceps, son buenas intervenciones para evitar la recidiva de la flexión. En casos más benignos una buena intervención consiste en el descenso de la tuberosidad ante rior de la tibia con el tendón rotuliano, operación de Chandler. 
Adducción de cadera: Cuando fracasen las medidas profilácticas o los métodos de corrección incruenta, se recurrirá en los casos en que no puede mantenerse la corrección, a la resección de las dos ramas del nervio sbturador; en cambio, cuando por métodos incruentos no puede obtenerse la corrección. la conducta varía para los casos benignos y para los severos; en los primeros, sección del recto interno y del gran adductor y resección de la rama anterior del nervio obturador; para los cascs severos tenotomía de recto interno, grande y pequeñns adductores, más resección de ambas ramas del nervio obturadcr.

Flexión de cadera: Operación original de Soutter o la modificación de Campbell.

Miembro supetior. - Las situaciones son más difíciles de resolver por la fineza que requieren los movimientos de la mano: será necesario extremar la insistencia en la aplicación de los métodos de reeducación.

Las intervenciones quirúrgicas tienen aquí menos indica. ciònes aún que en el miembro inferior; según las circunstanciss y las necesidades, podrá recurrirse al trasplante de flexores (gran palmar y cubital anterior), a los extensores para corregir la flexión del puño; a una artrodesis de la parte externa de la articulación del puño y siempre que al paciente no le sea necesaria la flexo extensión de la radio carpiana para efectuar la fexo extensión de los dedos; a un trasplante de cubital anterior por el dorso a la extremidad inferior del radio para corregir una pronación exagerada del antebrazo, etc.

Las intervenciones en codo y brazo son todavía de aplicacićn más excepcional aún.

E1 tratamiento quirúrgico de la lordosis y las escoliosis tienen indicaciones excepcionalísimas.

Cuando exista luxación unilateral de cadera podrá recurrirse a la artrodesis mixta intra y extra articular. que es una intervención que puede reportar resultados muy satisfactorios.

Cualquiera sea el tipo de intervención a que se recurra. para obtener buenos resultados y consolidarlos. será siempre necesario insistir en el tratamiento reeducador.

Las intervenciones sobre el sistema nervioso, ramisección introducida por Royle; la operaciór de Foerster, sección de las raíces posteriores: las cordotomias son intervenciones que ha. cen correr grandes riesgos al paciente, al mismo tiempo que dé resultados inseguros y discutibles. 


\section{B) Parálisis obstétricas.}

Encararemos exclusivamente el tratamiento de lạs parálisis obstétricas verdaderas, dejando absolutamente de lado todas otras lesiones capaces de dar clínicamente el "Sindrome de parálisis obstétrica radicular del miembro superior". tales cemo los decolamientos de la epífisis humeral superior. las fracturas de clavícula: etc.

Desde el punto de vista del tratamiento, las parálisis obs. tétricas deben ser encaradas en dos inomentos distintos:

A) En el recién nacido durante los tres primeros meses;

B) En el enfermo con la secuela constituída.

A) Recién nacido y tres primeros meses. - Pueden presentarse dos eventualidades:

$1 .{ }^{\circ}$ Sin gran contractura que haga difícil la corrección manual simple de la actitud viciosa (en el recién nacido en general no habrá ninguna contractura importante);

2. Con contractura grande que impide la cortección manual de la actitud viciosa (el tratamiento de este sub-grupo entra en el del grupo B).

Nos limitaremos aquí, por consiguiente, solamente al tratamiento del sub-grupo $1 .{ }^{\circ}$. En estos casos, el tratamiento en sus líneas generales oscila dentro de las siguientes tendencias:

a) Quirúrgica, luego de un tratamiento ortopédico conservador de espera, que más adelante describiremos. y si las lesicnes no mejoran dentro de un plazo de unos 45 dias. La intervención consiste en el abordaje del plexo, la extirpación del te jido de cicatriz y la sutura de los troncos desgarrados.

Los grandes partidarios de las intervenciones han sido los cirujanes norteamericanos y decimos han sido. poraue actualmente ha pasado el periodo de auge de estas intervenciones; on realidad no podia ser de otra manera. dado que la sutura de las raices nerviosas prácticamente no se acompaña de éxitos y el tejido de cicatriz extirpado casi siempre es sustituído por nuevo tejido de cicatriz.

b) Tendencia electroterápica, métodos de eficacia discatible. aplicados siempre durante los períodos de mejorías o tecuperaciones espontáneas, siempre se hace constar en su activo 10 que en realidad es el resultado del proceso de reparación natural. En materia de parálisis obstétrica no creemos en sus éxitos y afirmamos que a veces. los resultados son contrapro. ducentes. porque al exagerarse las contrac'uras, se agravan las acciludes viciosas y disminuye el valor funcional de los miembros lecionados. 
c) Tendencia ortopédica incruenta, combinación del uso de aparatos de posición. masajes, movilización activo-pasiva, recomendado por gran número de autores, su técnica ha sido reglada por Harry Platt.

En primer lugar, el paciente usará una férula de posición que mantiene su brazo en $90^{\circ}$ de rotación interna y $90^{\circ}$ de abducción, codo en semi-f́lexión, antebrazo en supinación, mano en posición indiferente o en flexión dorsal, según los casos: esta posición procura reposo al plexo braquial, favorece el proceso de reparación espontánea al evitar la distensión secundaria de las raíces bajo la influencia del peso del miembro y se opone a la constitución de actitudes viciosas. Después de la primera semana, se instituyen dos sesiones diarias de masaje y movilización pasiva suave, dejándose más adelante el miembro en libertad una o dos horas por día, para que el paciente efectúe movilización activa. Progresivamente se prolongan los periodos de movilización activa y luego el paciente solamente usa el aparato de posición durante la noche; esto último debe, a veces, ser hecho durante años, siempre que exista la más mínima tendencia a la rotación interna del brazo. Más adelante. se agregan verdaderos ejercicios gimnásticos localizados al miembro que ha sido el asiento de la parálisis obstétrica y de acuerdo con la mejoría experimentada.

B) En el enfermo con su secuela ya constituída haremos dos sub-grupos para simplificar las situaciones:

a) Tratamiento de las deformaciones;

b) Tratamiento de la secuela paralítica definitiva.

a) Tratamiento de las deformaciones: Métodos incruentos y métodos cruentos.

Métodos incruentos: Anestesia general, corrección de la actitud viciosa, yeso tóraco braquial, manteniendo la corrección durante un par de meses. Aplicable a niños hasta de dos o tres años, según las circunstancias. Luego, aparato amovible para mantener su miembro corregido y tratamiento fisioterápico a base de calor, masaje y movilización activo-pasiva.

Métodos cruentos: Tenotomia del supra-escapular, capsulotomía, alargamiento del tendón del gran pectoral, del corto bíceps y el tóraco braquial, según las necesidades, para cortegir la rotación interna del brazo: tenotomía del redondo pronador para suprimir la pronación. La rotación interna del brazo también puede ser corregida por una osteotomia.

Yeso tóraco braquial durante 25 días, en caso de sección exclusiva de partes blandas, entre 45 y 60 dias, en caso de 
una osteotomía, luego aparato amovible y complemento fisioterápico.

b) Tratamiento de las secuelas paralíticas definitivas: Plantearemos esquemáticamente tres eventualidades: 1) Pérdida de la extensión de puño y dedos, una excelente intervención es el trasplante de fuerzas flexoras (gran palmar, cubital anterior) al dorso, a los extensores; 2) Impotencia solamente parcial de la flexión del codo, operación de Steindler. llevar la inserción de los músculos epitrocleares que son flexores accesorios más hacia arriba en la cara anterior del húmero, transformándolos asi en flexores de mayor potencia; 3 ) Pérdida de la abdueción del hombro con buen trapecio, artrodesis del hombro que en el conjunto da mejores resultados que el trasplante de trapecio mediante un tendón de fascia lata a la $\mathrm{V}$. deltoídea. Como complemento indispensable, precediendo y siguiendo a los tiempos operatoríos: termoterapia. masaje, movilización pasiva y sobre todo movilización activa, reeducación mantenida de manera tenaz y persistente.

\section{C) Parálisis infantil.}

Tema de gran extensión. de gran complejidad, dada la multiplicidad de matices, en este co-relato forzosamente linitado, sólo podremos rozar el tema en sus directivas de orden general. Insistiremos, esc sí, sobre un aspecto que a primera vista choca un poco al espíritu de quien desconoce el problema $y$ es la importancia fundamental que en esta afección tiene el tratamiento ortopédico, no solamente en su período de secuelas, sino desde la iniciación del cuadro paralítico desde el mismo periodo agudo, desde el momento mismo en que aparecen las parálisis, entendiendo por tal, no solamente la parálisis total y absoluta, sino también todo grado dilucidable de disminución de la fuerza del músculo.

La parálisis infantil es una afección que desde los puntos de vista de su estudio clínico, fisiopatológico y tratamiento de las lesiones del aparato del movimiento en sus etapas agudas, sub-aguda, convalecencia y crónica o de secuela. pertenecen al dominio de la ortopedia. Dividiremos su tratamiento en dos etapas:

A) Primera etapa: Entendemos por tal, desde el poriodo agudo inclusive y durante la convalecencia hasta dos o tres años del debut:

B) Etapa crónica o de secuela. 
A) Primera etapa: El tratamiento durante este petiodo tiene importancia fundamental para el porvenir del paciente.

Conocida es por todos la imposibilidad de evitar la instalación de las parálisis, porque los tratamientos preventivos han fracasado, las tentativas de inmunización activa o pasiva son ineficaces. Tampoco existe ningún método que permita tratar la lesión de las células nerviosas una vez que la afección estalló, la pretendida sueroterapia curativa también ha sido un fracaso.

Si no se puede evitar la instalación de las lesiones, si es luego imposible mejorar la localización nerviosa, la única solución actual está en reducir al minimo posible las manifes. taciones patológicas del aparato locomotor, aprovechando ta regresión o mejoría espontánea que siempre existe y esta finalidad se logra por los siguientes recursos terapéuticos:

1. Reposo;

2. Protección de los músculos y articulaciones;

3. Tratamiento fisioterápico.

Repose y protección de los músculos y arriculaciones; constituyen el tracamiento crtopédico precoz que se instituye en los períodos paralíticos agudo y sub-agudo, desde que las parálisis aparecen.

El reposo local que va unido a la protección de los múscuJos y articulaciones se efectúa en férulas livianas y en posición de reposo neutro, de completo relajamiento muscular de él o los miembros afectados.

Esta parte del tratamiento tiene por objeto evitar a los músculos los estiramientos y distensiones que disminuyen sa vitalidal y su valor funcional, al mismo tiempo que se hace la prcfilaxis desde el comienzo, de las actitudes viciosas. Ese período de reposo dura entre dos semanas y tres meses a partir del debut.

Tratamiento fisioterápico. - Se agrega en la convaleconcia (luego de desaparecida la fiebre, los dolores espontáneos y la sensibilidad muscular a la presión, a las dos semanas o a los tres meses del debur.

Comprende: 1. calor; 2 masaje; 3. movilización pasiva; 4. ejercicio muscular activo.

Calor y masaje suave y corto para aumentar el régimen circulatorio del músculo; movilización pasiva suave durante las primeras etapas y efectuada con la suavidad y restricción necesarias para no estirar los músculos lesionados; ejercicio muscular activo que es el gran estimulante de la contractilidad 
muscular, mejor que todos los tratamientos electroterápicos y que debe ser electivo, localizado, graduado y evitando la fatiga que en los músculos poliomielíticos es fácil de alcanzar.

Este tratamiento de la primera etapa que aprendimos en Estados Unidos de Norte América, lo hemos ensayado entr? nosotros con motivo de nuestra última epidemia de 1941; sus resultados son ampliamente satisfactorios, superiores a todos los tratamientos con electroterapia.

B) Etapa crónica o de secuélas. - Es un tratamiento ortopédico quirúrgico fisioterápico asociado. En esta etapa de secuelas, el tratamiento tiene dos indicaciones que a veces se realizan simultáneamente: a) corregir deformaciones; b) manrener la corrección obtenida, evitando la reproducción de la deformación y buscar la recuperación funcional. Esquemáticamente estos fines se alcanzan recurriendo a los siguientes mét:dos terapéuticos:

Tratamiento de la deformación constituida, su corrección: Métodos incruentos; yesos en varias etapas con o sin narcosis, método del torniquete, rápidos $\mathrm{y}$ con narcosis los osteoclastos. lento y gradual método de Mommsen. Métodos iruentos: actuando sobre partes blandas y respetando los músculos restantes, alargamientos plásticos, alargamientos artificiales con seda o aponeurosis, transposición de inserciones: actuando sobre partes blandas y prescindiendo de los músculos restantes, secciones musculares, aponeuróticas, tendinosas y capsulares; actuando sobre partes óseas y osteoarticulares. osteotomías para articulares y resecciones osteoplasticas.

Mantener Ia corrección obtenida, evitar la reproducción de la deformación: Provisoriatnente por aparatos ortopédicos, yesos, celuloide, aluminio, etc.

Definitivamente y activamente buscando restablecer el equilibrio muscular y la función articular por intermedio de transplantes musculares, transplantes tendinosos. transplantes nerviosos. neurotización de los músculos paralizados: definitivamente $y$ pasivamente por la limitación o abolición de la tunción articular, valiéndonos de artrodesis, artrorisis, tenodesis. fasciodesis, construcción de ligamentos artificiales.

Se comprenderá que dada la extensión de este capítulo, no podemos hacer otra cosa que dejario esbozado.

Como se habrá podido apreciar a través de esta sucinta exposición, la Ortcpedia tiene una importancia y un alcanca fundamentales para el tratamiento de estas afecciones nerviosas de la infancia. 